\title{
Isometric immersions, energy minimization and self-similar buckling in non-Euclidean elastic sheets
}

\begin{tabular}{|l|l|}
\hline Item type & Article \\
\hline Authors & $\begin{array}{l}\text { Gemmer, John; Sharon, Eran; Shearman, Toby; } \\
\text { Venkataramani, Shankar C. }\end{array}$ \\
\hline Citation & $\begin{array}{l}\text { Isometric immersions, energy minimization and self- } \\
\text { similar buckling in non-Euclidean elastic sheets 2016, 114 } \\
(2): 24003 \text { EPL (Europhysics Letters) }\end{array}$ \\
\hline Eprint version & Final accepted manuscript \\
\hline DOI & 10.1209/0295-5075/114/24003 \\
\hline Publisher & $\begin{array}{l}\text { EPL ASSOCIATION, EUROPEAN PHYSICAL } \\
\text { SOCIETY }\end{array}$ \\
\hline Journal & EPL (Europhysics Letters) \\
\hline Rights & Copyright @ EPLA, 2016 \\
\hline Downloaded & 18-Jul-2016 22:58:05 \\
\hline Link to item & http://hdl.handle.net/10150/617197 \\
\hline
\end{tabular}




\title{
Isometric immersions, energy minimization and self-similar buck- ling in non-Euclidean elastic sheets.
}

\author{
John Gemmer ${ }^{1,5}$, Eran Sharon ${ }^{2,5}$, Toby Shearman ${ }^{3}$ and Shankar C. Venkataramani ${ }^{3,4,5}$ \\ 1 Division of Applied Mathematics, Brown University, Providence, RI 02906, USA \\ 2 Racah Institute of Physics, The Hebrew University, Jerusalem, 91904, Israel \\ 3 Program in Applied Mathematics, University of Arizona, Tucson, AZ 85721, USA \\ 4 Mathematics Department, University of Arizona, Tucson, AZ 85721, USA \\ 5 Kavli Institute of Theoretical Physics, University of California, Santa Barbara, CA 93106, USA
}

$\begin{array}{ll}\text { PACS } & 46.70 . \mathrm{Hg} \text { - Membranes, rods and strings } \\ \text { PACS } & 46.32 .+\mathrm{x} \text { - Static buckling and instability } \\ \text { PACS } & 02.40 . \mathrm{Hw} \text { - Classical differential geometry }\end{array}$

\begin{abstract}
The edges of torn plastic sheets and growing leaves often display hierarchical buckling patterns. We show that this complex morphology (i) emerges even in zero strain configurations, and (ii) is driven by a competition between the two principal curvatures, rather than between bending and stretching. We identify the key role of branch-point (or "monkey-saddle") singularities in generating complex wrinkling patterns in isometric immersions, and show how they arise naturally from minimizing the elastic energy.
\end{abstract}

Introduction. - The rippling patterns observed in torn plastic sheets [1-3], leaves [4-7] and swelling hydrogels [8-10] provide striking examples of periodic and selfsimilar patterns; see fig. 1. Within the formalism of finite elasticity, such patterns are understood as resulting from the sheet buckling to relieve growth induced residual strains $[11,12]$. On the one hand, complex, self-similar patterns in elastic sheets can arise from boundary conditions that preclude the possibility of relieving in-plane strains [13-15]. On the other hand many growth patterns generate residual in-plane strains which can be entirely relieved by the sheet forming an isometric immersion of a hyperbolic Riemannian metric; indeed smooth hyperbolic metrics on bounded domains can always be immersed in $\mathbb{R}^{3}$ by smooth isometries [16] (but not so for unbounded domains [17-19]). Why then do we observe self-similar buckling patterns in free elastic sheets? In this letter we report on multiple strands of recent work, that, taken together, address this puzzle.

We show that a large class of growth profiles admit smooth (i.e. infinitely differentiable) as well as (many) non-smooth configurations of the sheet with vanishing inplane strain. The non-smooth configurations are piecewise surfaces constructed by gluing together isometries along "lines of inflection" and at "branch points" in such a man-
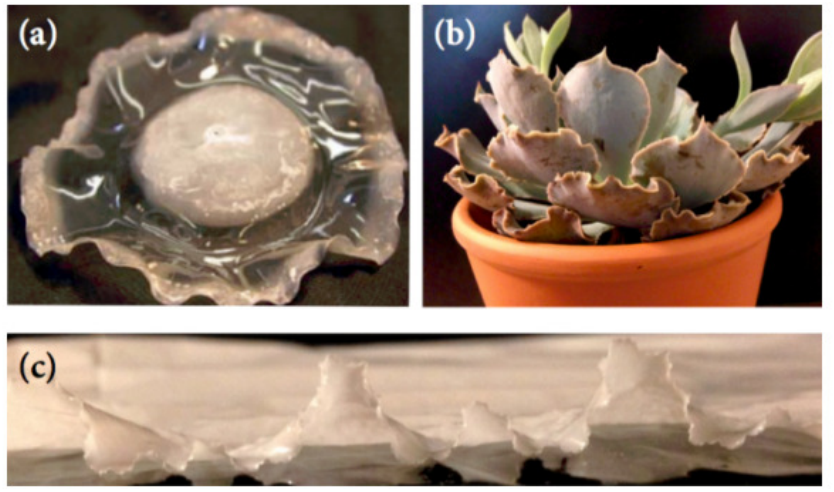

Fig. 1: Examples of complex wrinkling patterns in swelling thin elastic sheets. (a) Hydrogel disk with non-uniform swelling pattern. (b) Ornamental echeveria house plant. (c) Edge of a torn trash bag.

ner that the resulting surface does not concentrate bending energy. We also show that minimizing the bending energy among the various isometric immersions naturally leads to complex, self-similar, wrinkling patterns.

Non-Euclidean model of elasticity. - The nonEuclidean formalism of thin sheet elasticity [20] posits 
that growth permanently deforms the intrinsic distance between material points, so growth is encoded in a Riemannian metric g. Specifically, material points on the center surface are labelled by coordinates $(x, y) \in \Omega$, a subset of $\mathbb{R}^{2}$, and the distance between such points is given by the arc-length element:

$$
d s^{2}=g_{11}(x, y) d x^{2}+2 g_{12}(x, y) d x d y+g_{22}(x, y) d y^{2} .
$$

By the Kirchhoff hypothesis [21], the conformation of the sheet as a 3 -dimensional object in $\mathbb{R}^{3}$ is determined by an immersion $F: \Omega \rightarrow \mathbb{R}^{3}$ of the center surface. The equilibrium configuration is then taken to be a global minimizer of an elastic energy modeled as the sum of stretching and bending contributions:

$$
\begin{aligned}
E[F] & =\mathcal{S}[\gamma]+t^{2} \mathcal{B}[H, K] \\
& =\int_{\Omega} Q(\gamma) d x d y+t^{2} \int_{\Omega}\left(4 H^{2}-2 K\right) d x d y,
\end{aligned}
$$

where $\gamma=(\nabla F)^{T} \cdot \nabla F-\mathbf{g}$ denotes the in-plane strains in the center surface, $t$ is the thickness of the sheet, $Q$ is a quadratic form, and $H$ and $K$ are respectively the mean and Gaussian curvatures of the center surface [20, $22]$. Provided they exist, the $t \rightarrow 0$ (vanishing thickness) limits of minimizers of the elastic energy (2) are necessarily finite bending energy isometric immersions [23].

For isometries, the metric determines the Gauss curvature $K=\kappa_{1} \kappa_{2}$, where $\kappa_{1,2}$ are the principal curvatures [16]. For a fixed Gauss curvature, decreasing $\left|\kappa_{1}\right|$ increases $\left|\kappa_{2}\right|$ and vice-versa so there is a competition between their contributions to the bending content $\int\left(\kappa_{1}^{2}+\kappa_{2}^{2}\right) d x d y$. We define a geometric quantity, the disparity $\eta$, by:

$$
\eta \equiv \frac{H}{\sqrt{|K|}}=\frac{1}{2}\left(\sqrt{\left|\frac{\kappa_{1}}{\kappa_{2}}\right|}-\sqrt{\left|\frac{\kappa_{2}}{\kappa_{1}}\right|}\right)
$$

The bending energy density $\kappa_{1}^{2}+\kappa_{2}^{2} \sim H^{2}=\eta^{2}|K|$. Thus $\eta$ quantifies the local contribution to the bending content from the mismatch in the principal curvatures.

Power law metrics and single wavelength isometries. - We investigate finite bending energy isometric immersions of the strip $\Omega=\mathbb{R} \times[0, W]$ with the metric

$$
\mathbf{g}=\left(1+2 \epsilon^{2} f(y)\right) d x^{2}+d y^{2}, \quad f(y)=\frac{\alpha}{4(\alpha+1)}\left(1+\frac{y}{l}\right)^{-\alpha}
$$

where $\alpha \in(0, \infty), l$ is a length scale and $\epsilon>0$. These metrics corresponds to $y$ dependent growth in the $x$ direction localized near the $y=0$ edge of the sheet. It includes the metrics considered in $[2,3,6,7,15]$ as particular cases.

For $\epsilon \ll 1$, approximate isometries are obtained from the Föppl - von Kármán ansatz $F(x, y)=\left(x+\epsilon^{2} u, y+\right.$ $\left.\epsilon^{2} v, \epsilon w\right)$. In the small slope regime, i.e. for $\epsilon \ll 1$, the mean and Gaussian curvatures are given by $H=\frac{\epsilon}{2} \Delta w$ and $K=\epsilon^{2} \operatorname{det}\left(D^{2} w\right)$ [24]. If $w$ satisfies

$$
\operatorname{det}\left(D^{2} w(x, y)\right)=-f^{\prime \prime}=-\frac{\alpha^{2}}{4}\left(1+\frac{y}{l}\right)^{-\alpha-2}
$$

we can solve for $u$ and $v$ to obtain isometries up to $O\left(\epsilon^{2}\right)$ [24]. It is straightforward to check that eq. (5) has a one parameter family of product solutions

$$
w^{0}(x, y)=k^{-1} \psi(k x)(1+y / l)^{-\alpha / 2}
$$

where $\psi^{\prime 2}+|\psi|^{2 \alpha /(2+\alpha)}=1$. These product solutions are characterized by a single wavelength that is set by $k$ independent of $y$, so there is no refinement as $y \rightarrow 0$. The product solutions in eq. (6) have finite bending content for $\alpha>2 / 3$ and their mean curvature is given by

$$
H(x, y)=\epsilon\left[\frac{c_{1}(\xi)}{k l^{2}(1+y / l)^{\alpha / 2+2}}-\frac{c_{2}(\xi) k}{(1+y / l)^{\alpha / 2}}\right],
$$

where $\xi=k x$, and $c_{1,2}$ are positive functions, independent of $k$. Averaging $\epsilon^{-2} H^{2}$ in $x$ we obtain the $k$ dependence of $\bar{B}$, the normalized (by $\epsilon^{2}$ ), nondimensional, bending content per unit length in the $x$ direction:

$$
\bar{B} \sim C_{1} k^{2} l \int_{0}^{W} \frac{d y}{(1+y / l)^{\alpha}}+\frac{C_{2}}{k^{2} l^{3}} \int_{0}^{W} \frac{d y}{(1+y / l)^{\alpha+4}}
$$

with $C_{1}, C_{2}$ positive constants. The optimal "global" wavelength $\lambda_{\text {glob }}$ is given by:

$$
\lambda_{g l o b} \sim \frac{1}{k^{*}} \sim l\left|\frac{3-\alpha}{1-\alpha} \frac{(1+W / l)^{1-\alpha}-1}{(1+W / l)^{-3-\alpha}-1}\right|^{\frac{1}{4}},
$$

where $k^{*}$ is the value of $k$ that minimizes $\bar{B}$. We can also determine the optimal "local" wavelength $\lambda_{l o c}(y)$ by minimizing the average of $H^{2}$ at a given $y$, (eq. (8) without integrating in $y$ ) to obtain

$$
\lambda_{l o c}(y) \sim l(1+y / l)=(y+l) .
$$

This is related to the buckling wavelength for a thin strip cut out of the sheet $[3,7]$ at $y . \quad \lambda_{l o c}(0) \approx \lambda_{g l o b}$, but for $W \gg l$, there is a range of $y$ where $\lambda_{l o c}(y)$ differs substantially from $\lambda_{\text {glob }}$, giving an energetic impetus for the sheet to increase the buckling wavelength as one moves away from the edge at $y=0$.

Branch points in isometric immersions. - There is, however, a geometric obstruction to changing the wavelength in $y$. Smooth surfaces with negative Gaussian curvature are locally saddle-shaped. At every point $p$ on such a surface there are two asymptotic directions given by the intersection between the surface and its tangent plane at $p$. An argument, that we will present elsewhere, proves that surfaces that have precisely two asymptotic lines through every point will necessarily have a single wavelength and therefore a variation of the wavelength in $y$, i.e. sub-wrinkling, is prohibited. We introduce the notion of "branch point" singularities which allow for multiple asymptotic directions at points and thus circumvent the obstruction to sub-wrinkling in smooth isometries.

First, consider solutions of $\operatorname{det}\left(D^{2} w\right)=-1$, the smallslope approximation to the isometry condition for a surface with constant negative Gaussian curvature. Let $(x, y)$ 
and $(r, \theta)$ denote Cartesian and polar coordinates on $\mathbb{R}^{2}$. Piecewise solutions to $\operatorname{det}\left(D^{2} w\right)=-1$ are given by

$w(x, y)=a x+b y+c+ \begin{cases}y\left(x-y \cot \left(\theta_{+}\right)\right) & 0 \leq \theta \leq \theta_{+} \\ y\left(x+y \cot \left(\theta_{-}\right)\right) & -\theta_{-} \leq \theta \leq 0\end{cases}$

where $0<\theta_{ \pm}<\pi$. The surface $z=w(x, y)$ has a continuous tangent plane along the ray $\{\theta=0\}$ where the two "pieces" are attached, so bending energy does not concentrate on this ray. This ray is a line of inflection - a principal curvature is discontinuous across this ray, but the bending energy density is finite.

This construction can be extended to piecewise quadratic surfaces about any point $\left(x_{0}, y_{0}\right)$. Given $2 n$ (an even number) and a sequence of angles $\theta_{0}<\theta_{1}<\theta_{2}<$ $\ldots<\theta_{2 n}=2 \pi+\theta_{0}$, and a plane $z=a x+b y+c$, define lines of inflection on the plane by $x(\lambda)=x_{0}+\cos \left(\theta_{i}\right) \lambda$ and $y(\lambda)=y_{0}+\sin \left(\theta_{i}\right) \lambda$ with $\lambda \geq 0$. These lines determine quadratic surfaces with $K=-1$ that can be joined along these lines of inflection, generalizing our construction in ref. [25]; see fig. 2(a). The resulting surface is smooth only if $n=2, \theta_{2}=\theta_{0}+\pi$ and $\theta_{3}=\theta_{1}+\pi$. In particular, the surface is not smooth if $n \geq 3$. We define the point $\left(x_{0}, y_{0}\right)$ where the $2 n$ lines of inflection meet $(n \geq 3)$ to be a branch point or $n$-saddle for the surface; $\left(x_{0}, y_{0}\right)$ is indeed a branch point for the map $(x, y) \mapsto \nabla w$.

For a smooth quadratic surface, the tangent plane at a point $\left(x_{0}, y_{0}\right)$ intersects the surface along 4 rays with azimuthal angles $\varphi_{0}, \varphi_{1}, \varphi_{2}=\varphi_{0}+\pi, \varphi_{3}=\varphi_{1}+\pi$. We can introduce a branch point at $\left(x_{0}, y_{0}\right)$ using the tangent plane as the common plane, and define a daughter sequence of angles $\left\{\theta_{i}\right\}_{i=1}^{6}$ such that $\theta_{0}=\varphi_{0}<\theta_{1}<\theta_{2}<$ $\theta_{3}=\varphi_{1}<\theta_{4}=\varphi_{2}<\theta_{5}=\varphi_{3}<\theta_{0}+2 \pi$. Multiple branch points can introduced on the surface recursively as illustrated in fig. 2(b-c). Surfaces with multiple branch points can display sub-wrinkling or refinement - in fig. 2 the "number of waves" increases with radius. The network of asymptotic lines in figs. 2(a-b) are the asymptotic nets for the underlying surfaces [26].

We now consider the energy of surfaces with branch points. In the sector $\theta_{i-1} \leq \theta \leq \theta_{i}$, the disparity $\eta$ (defined in eq. (3)) is given by $\eta= \pm \cot \left(\theta_{i}-\theta_{i-1}\right)$. The sequence $\left\{0, \frac{3 \pi}{4}, \pi, \frac{7 \pi}{4}, 2 \pi\right\}$ and the daughter sequence $\left\{0, \frac{\pi}{4}, \frac{\pi}{2}, \frac{3 \pi}{4}, \pi, \frac{7 \pi}{4}, 2 \pi\right\}$ are unique in that all the sectors have disparity $\eta= \pm 1$, and thus have the same energy density. These sequences are therefore natural candidates for the local structure at bifurcations in energy minimizers corresponding to adding branch points.

Branch points in the strip geometry. - We now return to the strip geometry in eq. (4); we want to create patterns which have an effective wavelength that increases with $y$, so they have lower energy than the single wavelength isometry. A natural idea is to introduce branch points into the product solutions of eq. (5) to allow for refinement of the wavelength near $y=0$. However, the product solutions are not ruled by straight lines, and it is not obvious how one adapts the above construction.
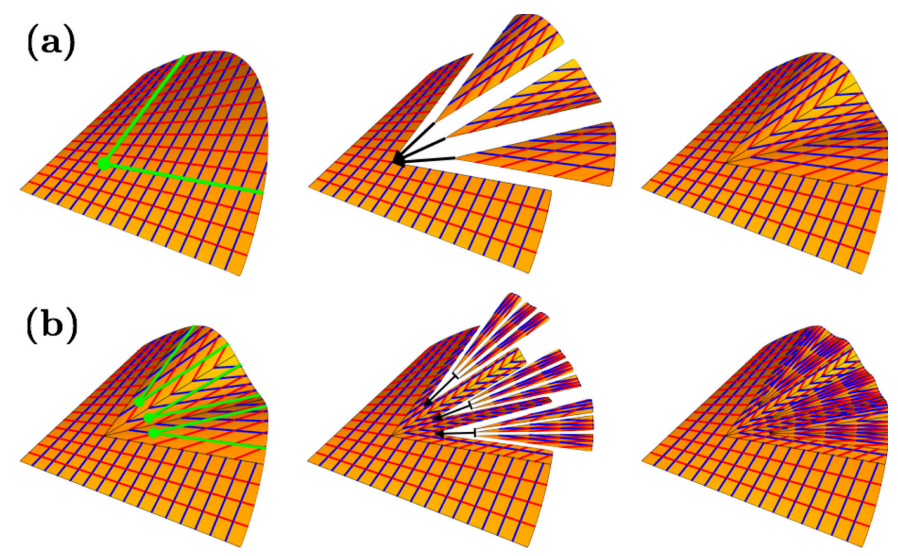

(c)

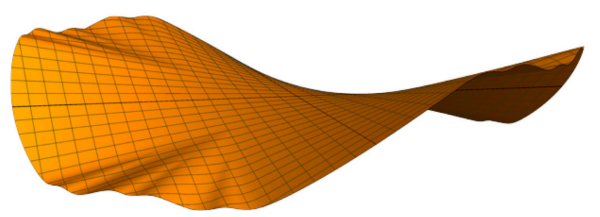

Fig. 2: Solutions to $\operatorname{det}\left(D^{2} w\right)=-1$ with branch points. (a) Three sub-wrinkle solution on the first quadrant. (b) Nine subwrinkle solution. (c) Extension to 36 sub-wrinkle solution on the plane.

We can construct solutions of eq. (5) with branch points as follows. Let $Z(X, Y)$ be a (piece of a) surface satisfying $Z_{X X} Z_{Y Y}-\left(Z_{X Y}\right)^{2}=-1$ with $Z_{X X} \neq 0, Z_{Y Y} \neq 0$ (true for any surface by rotating the $X Y$-axes if necessary). Define $P=\partial_{X} Z, Q=\partial_{Y} Z, \sigma=\left(f^{\prime \prime}(y)\right)^{1 / 4}$. Direct computation shows that the over-determined system

$$
\begin{aligned}
& \partial_{Y} y=-\sigma^{-2}, \quad x=\sigma^{-1}\left(X+\xi_{+}+\xi_{-}\right), \\
& \partial_{x} w=P, \quad \partial_{y} w=\sigma\left(Q+\xi_{+}-\xi_{-}\right),
\end{aligned}
$$

satisfies $\partial_{y}\left(\partial_{x} w\right)=\partial_{x}\left(\partial_{y} w\right), \operatorname{det}\left(D^{2} w\right)=-f^{\prime \prime}(y)$ if

$$
2 \sigma\left(\partial_{Y} \mp \partial_{P}\right) \xi_{ \pm}=\left(\partial_{Y} \sigma\right)\left(X \mp Q+2 \xi_{\mp}\right),
$$

and $\operatorname{det}\left(D^{2} Z\right)=-1$. Solving eq. (12) reduces to integrating ODEs on surfaces satisfying $\operatorname{det}\left(D^{2} Z\right)=-1$. This procedure maps the asymptotic net of the surface $Z(X, Y)$ into that of the surface $w(x, y)$ and thus maps branch points to branch points. Consequently there exist many non-smooth solutions to (5) for metrics with $y$-dependent growth. In particular eq. (11) yields $(1+y / l) \sim Y^{-2}$ for the metric in ref. [2]; in this case, refinement with increasing $Y$ for $Z(X, Y)$ corresponds to sub-wrinking as $y \rightarrow 0$.

Instead of using eqs. (11)-(12), we directly generate discrete asymptotic nets [26] (allowing for potential branch points) that solve (a discretization of) eq. (5) - it is easier to impose boundary conditions in the latter approach. Figure 3(a) illustrates a "discrete isometric immersion" obtained by introducing branch points into the product solution of eq. (5). As the step size of the discretization goes to zero, the asymptotic net converges, by construction, to 
a surface with a continuously varying tangent plane, i.e. no ridges or cone-points. As expected from eqs. (11)-(12), our numerical algorithm generates a quad-mesh with the same topology as the meshes in fig. 2(b). Figure 3(b) demonstrates the convergence of the asymptotic net to an isometry of the metric in (4) with $\alpha=1$ by comparing the (nondimensional) "target curvature" $K(y)=-f^{\prime \prime}(y)$ with the discrete Gaussian curvature $K_{d}$ of the asymptotic net.

\section{(a)}

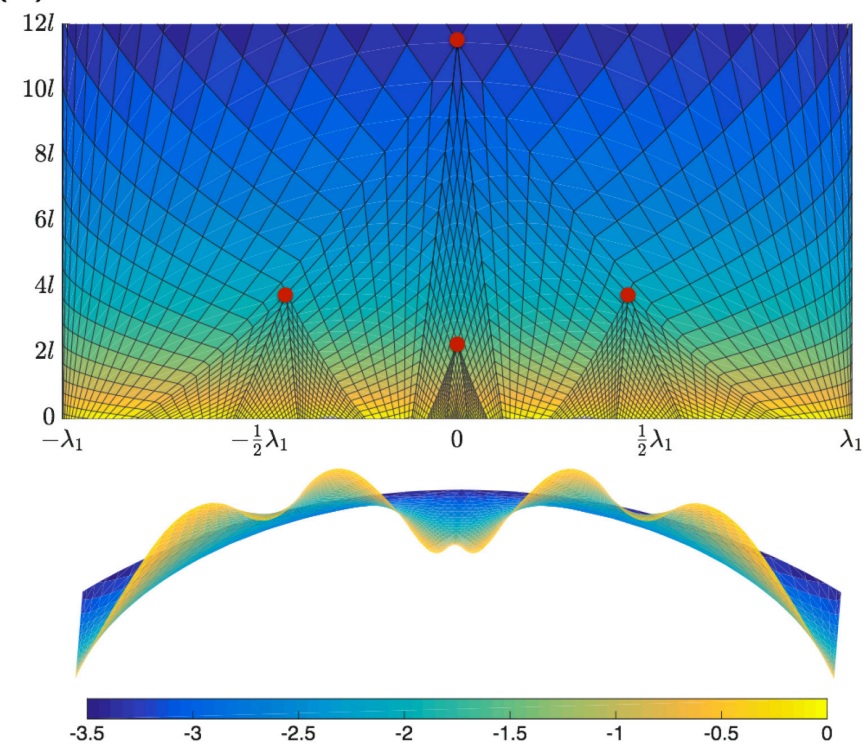

(b)

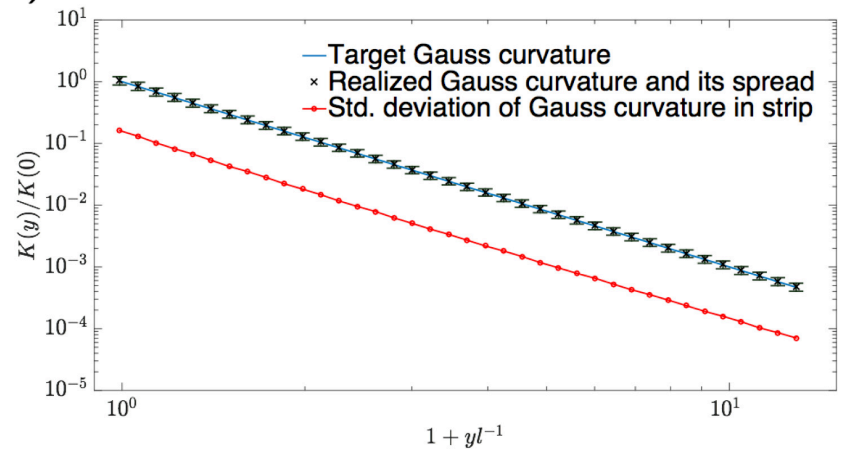

Fig. 3: (a) Discrete approximation of a four branch point (highlighted) isometric immersion for growth profile $f(y)=$ $(1+y / l)^{-1}$. The surface is colored by $\log _{10}\left(K_{d} / K(0)\right)$, the logarithm of the discrete curvature; note that the curvature (essentially) only depends on $y$. (b) Mean and spread of the discrete Gaussian curvature on thin "horizontal" strips.

Solutions $w(x, y)$ of eq. (5) can be rescaled $-\tilde{w}(x, y)=$ $s_{1}^{-2} s_{2}^{\alpha} w\left(s_{1} x, s_{2}(y+l)-l\right)$ is another solution for scale factors $s_{1}>0, s_{2} \geq 1$. Figure 4(a) shows the minimum energy among the 2-parameter family of isometries obtained by rescaling the solution shown in fig. 3. As $W / l$ increases, it is indeed energetically favorable to introduce branch points in $0 \leq y \leq W$; fig. 4(b) illustrates that the (a)
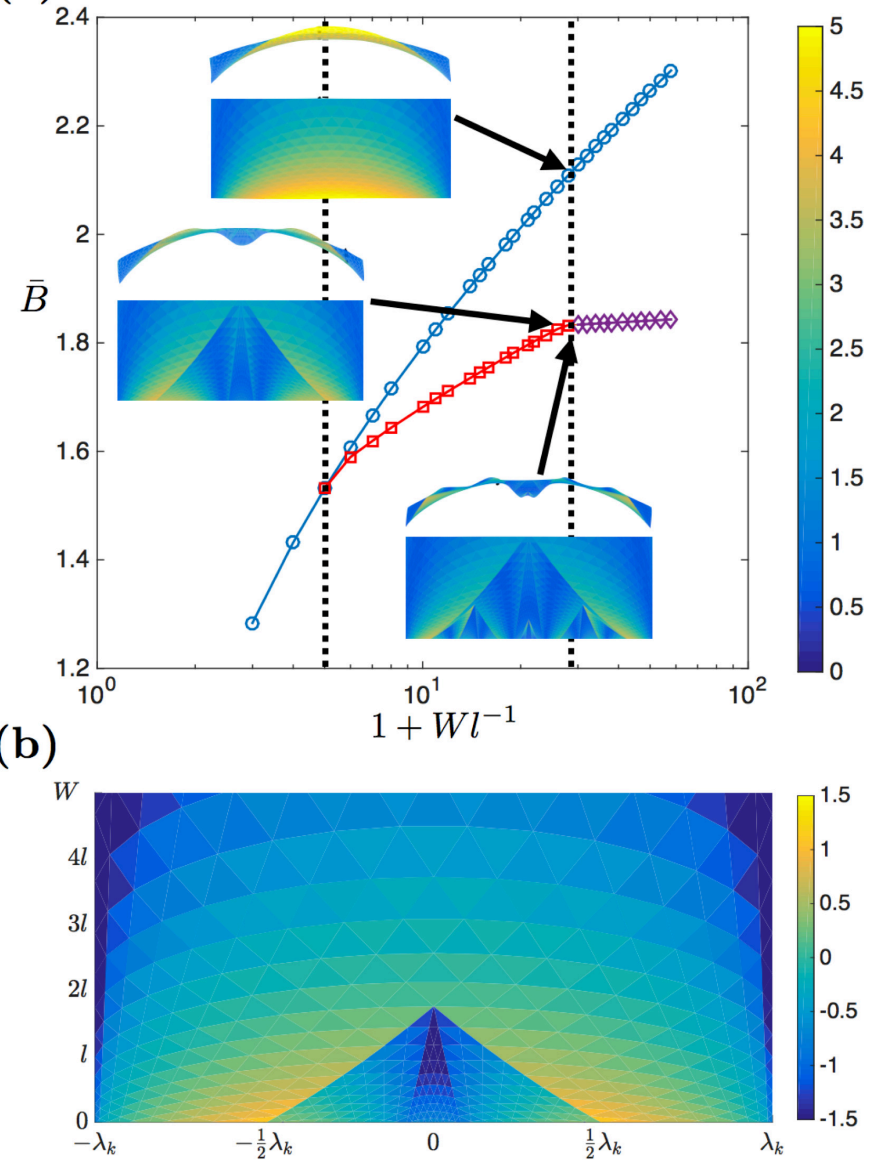

Fig. 4: (a) Bending energy minimized over a 2-parameter family of isometries for $f(y)=(1+y / l)^{-1}$. Circles (blue) correspond to single wavelength isometries, squares (red) to surfaces with one branch point, diamonds (magenta) to surfaces with four branch points. The insets are colored by the normalized energy density $\epsilon^{-2} l^{2}\left(4 H^{2}-2 K\right)$. (b) The disparity $\eta$ for the energy minimizing solution with $1+W l^{-1}=6$. This figure is to scale. The optimal values are $s_{1} \approx 9.1, s_{2} \approx 4.9, \lambda_{k} \approx 5.1 l$.

first branch point appears when $\eta \simeq 1$ at $(0,0)$. The insets in fig. 4(a) illustrate the divergence of $H$ with increasing $W / l$ for single wavelength isometries; they also show that introducing branch points does lower the bending energy.

Exponential metrics and singular edges. - As a special case we now consider the metric:

$$
\mathbf{g}=\left(1+\epsilon^{2} \exp (-2 y / l)\right) d x^{2}+d y^{2}
$$

on the "positive" half-plane $\mathbb{R}_{+}^{2}=\mathbb{R} \times[0, \infty)$, and also on all of $\mathbb{R}^{2}$. Equation (5) has a one parameter family of solutions $w^{0}(x, y)=\sqrt{2} k^{-1} \cos (k x) \exp (-y / l)$. For $k=l^{-1}$, $w^{0}(x, y)$ is a harmonic function on $\mathbb{R}^{2}$ and $H=0$ (a minimal surface) in the small-slope approximation. Consequently, there is no impetus in the small-slope regime for sub-wrinkling. The metric (13) provides a natural setting to characterize the relevance of nonlinear effects in ex- 
act (i.e non small-slope) isometries on wrinkling patterns. We can find analytic isometries of the metric by power series expansions: $(x, y) \mapsto\left(x+\epsilon^{2} u, y+\epsilon^{2} v, \epsilon w\right)$ where $u=\sum_{i=0} \epsilon^{2 i} u^{i}, v=\sum_{i=0} \epsilon^{2 i} v^{i}$ and $w=\sum_{i=0} \epsilon^{2 i} w^{i}$, and requiring that the configurations have zero strain at each order in $\epsilon$. To extract useful information we need to resum the series; to this end we use methods based on Padé approximants [27]. The series solution does not converge on all of $\mathbb{R}^{2}$. Rather it converges on a region of the form $y>\Pi(x, \epsilon)$. The curve $y=\Pi(x, \epsilon)$ represents the singular edge [17], which is a horizon or limit to the extension of an isometry that agrees with $w^{0}(x, y)$ as $y \rightarrow \infty$.

With increasing $\epsilon$, the singular edge will first intersect with the half-plane $\mathbb{R}_{+}^{2}$ at $(0,0)$ (the maximum for $\left|\partial_{y y}^{2} w\right|$ ). If $\partial_{y y}^{2} w(0, y) \sim A\left(y+\epsilon-\epsilon_{0}\right)^{-(\beta+1)}$, we can infer $\epsilon_{0}$ and $\beta$ from the poles and residues of the Padé approximants to the logarithmic derivative $\partial_{y y}^{2} w(0,0) / \partial_{y} w(0,0)[27]$. In fig. 5 we plot the Dlog Padé approximants for the case $k l=1$ and find that $\epsilon_{0} \approx 2.86$ and $\beta=.500158$. This strongly indicates that a curvature diverges as $s^{-\frac{1}{2}}$, where $s$ is the distance to the singular edge. This divergence is consistent with the singular edge of the pseudosphere and other hyperbolic surfaces of revolution.

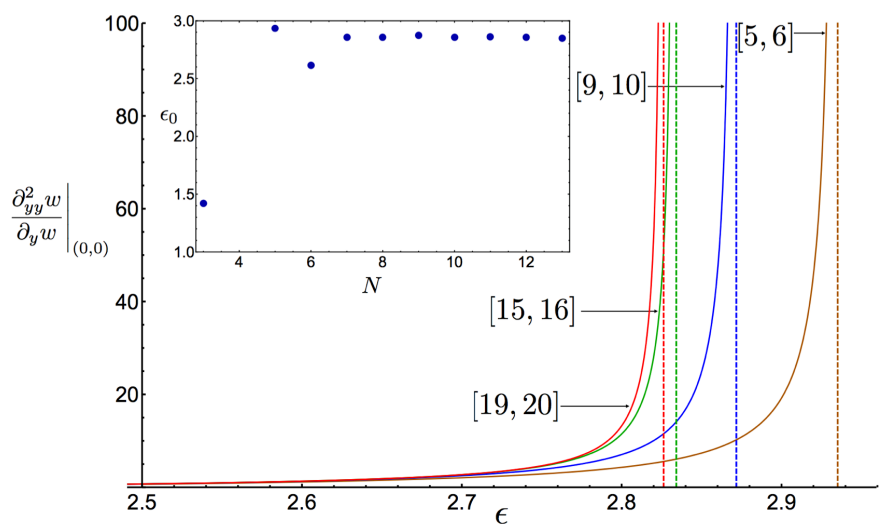

Fig. 5: $[N, N+1]$ Padé approximants to the logarithmic derivative $\partial_{y y}^{2} w / \partial_{y} w$ at $(0,0)$. The inset shows the value(s) of $\epsilon_{0}$ inferred from the vertical asymptote.

Figure 6 shows a contour plot of $\overline{\mathcal{B}}[\Delta w]=$ $(2 \pi l)^{-1} k \int_{0}^{2 \pi / k} \int_{0}^{\infty}(\Delta w)^{2} d y d x$ computed from the $[9,9]$ Padé approximant of $\Delta w$. The solid line indicates the $\epsilon$ dependent global wavelength $\lambda_{\text {glob }} \sim k^{-1}$ that minimizes $\overline{\mathcal{B}}$, a proxy for the bending energy per unit length.

For $\epsilon \ll 1$ the full elastic energy selects the expected wavelength from the small slope theory, i.e. $k=l^{-1}$, while for moderate values of $\epsilon \gtrsim 2$ the singular edge has a dramatic influence on the bending energy. The sheet can "move" the singular edge by a global decrease of the wavelength, i.e making $k$ larger. While this lowers the bending content near $y=0$, it will introduce large bending content in the rest of the sheet. As in the small-slope case, we expect that the minimizers of the elastic energy will instead locally refine the wavelength by introducing branch points near the edge $y=0$. The first branch point is expected to occur on the dash-dotted line in fig. 6 corresponding to the contour $H / \sqrt{|K|}=\eta=1$. The sub-wrinkling threshold for energy minimizers is at the intersection of the curves, i.e., $\epsilon \approx 1.75$. Accounting for in-plane displacement, this corresponds to a slope $\gamma=\left|\partial_{y} w\right| / \sqrt{1-\left(\partial_{y} w\right)^{2}} \approx 0.67$.

Complex buckling patterns can thus occur even in situations where the small-slope theory would predict no subwrinkling. Nonlinear contributions to in-plane strains are relevant for slopes as small as $\tan ^{-1}(\gamma) \approx 35^{\circ}$; this is consistent with the "real world" examples in fig. 1.

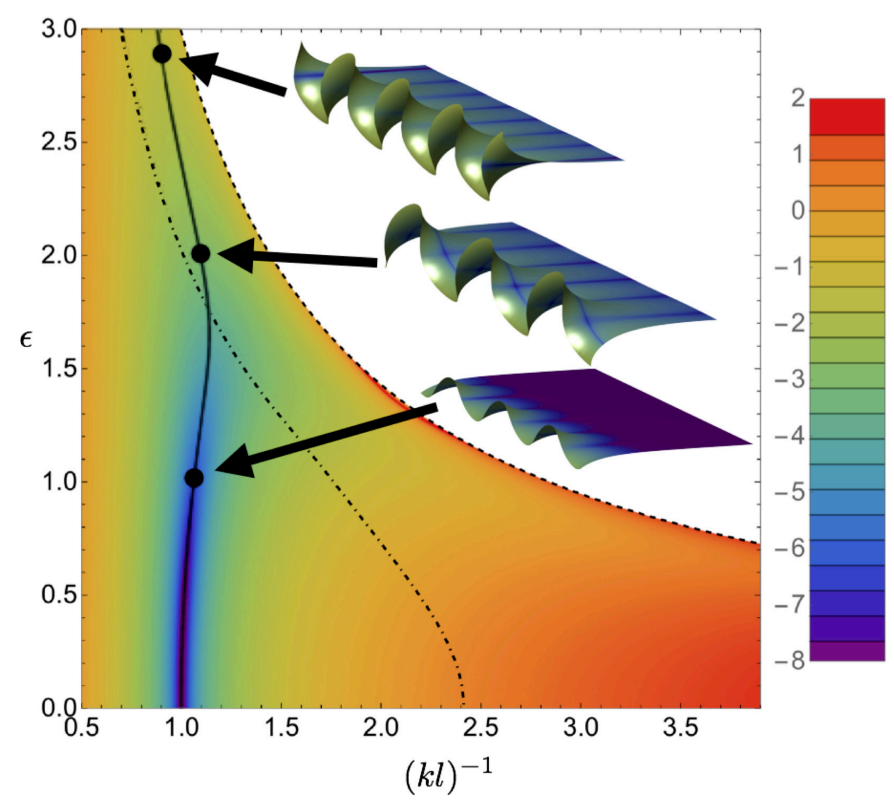

Fig. 6: Contour plot of $\ln (\overline{\mathcal{B}})$ as a function of the dimensionless wavelength $(k l)^{-1}$ and $\epsilon . \eta=1$ on the dash-dotted line and the solid line is the energy minimmizing wavelength. The dashed curve indicates values at which the singular edge touches $(0,0)$.

Discussion. - The existence of small-slope isometric immersions for hyperbolic free sheets with finite bending content ensures that the elastic energy per unit thickness scales as $t^{2}$. In the $t \rightarrow 0$ limit, this energy is much smaller than $t^{5 / 3}$, the energy scale for crumpled sheets [28, 29], $t^{4 / 3}$, the energy scale for sub-wrinkling non-Euclidean sheets subject to boundary conditions away from the wrinkled edge [15]. Free sheets therefore do not admit elastic ridges [29], $d$-cones [30] or wrinkles that balance stretching and bending energies $[3,15]$ because these defects cost too much energy; rather, the relevant singularities are branch points and lines of inflection. These defects are unique in that they do not concentrate elastic energy in the vanishing thickness limit. They arise for geometric reasons, viz. to bypass the geometric rigidity of smooth isometric immersions that prevents the refinement of the pattern wavelength. To our knowledge, this is the first example of a condensed matter system that is driven by geometric rather than energetic defects. 
We have shown that the buckling patterns in free sheets are a result of competition between the contributions to the bending energy from the two principal curvatures. Our key contribution is in identifying a mechanism, viz. the introduction of branch points/lines of inflection, that lowers the bending energy of the sheet while preserving the isometry constraint and the continuity of the tangent plane.

Audoly and Boudaoud [2] suggested the possibility that sub-wrinkling can arise in isometries. Their predicted scaling of the wavelength with the thickness implies that the limiting isometries have infinite bending content. The mechanism elucidated in this paper, viz. sub-wrinkling through introduction of branch points, is of an entirely different character, and outside the realm of the smooth truncated Fourier series solutions considered in [2].

We have analyzed our proposed mechanism and given a quantitative criterion for incipient sub-wrinkling in free sheets. Beyond the appearance of the first sub-wrinkle, the "far from threshold" behavior includes multiple branch points whose numbers/locations are determined by a "hard" problem - the global optimization of the bending energy. Our heuristic conclusions for this regime are that wide $(W \gtrsim l)$ sheets with an $O(1)$ variation in the logarithm of the Gaussian curvature $\log |K(y)|$ will generically manifest branch points and lines of inflection; fractallike, sub-wrinkling, profiles have lower energies than their single wavelength counterparts. Also, nonlinear contributions to the in-plane strain promote sub-wrinkling.

This work is primarily concerned with the vanishing thickness limit $t \rightarrow 0$. The results however are immediately transferable to physical sheets. In a physical sheet, the lines of inflection and branch points are replaced by boundary layers; the sheet configuration is indeed smooth, albeit with small scale structures. We expect that the smoothed defects are akin to the $t^{1 / 3}$ and $t^{1 / 2}$ boundary layers that resolve the non-smoothness of the $n$-saddle isometries of disks with $K=-1$ [31].

Our construction shows there are continuous families of low-energy states obtained by appropriately gluing together isometries. Variations within these families lead to "floppy modes" of deformation. Thin hyperbolic free sheets are thus easily deformed by weak stresses. The buckling pattern may be sensitive to the dynamics of the swelling process, experimental imperfections, or other external forces. This calls for a statistical description of the singularities and their interactions in this system.

We are very grateful to Benny Davidovitch and Tom Witten for useful discussions, and to the anonymous referees for their comments which significantly improved this paper. JG, ES and SV were supported by an US-Israel BSF grant 2008432. JG and SV were also supported by the NSF grant DMS-0807501. JG is currently supported by NSF-RTG grant DMS-1148284. This research was supported in part by the NSF Grant No. PHY11-25915.

\section{REFERENCES}

[1] Sharon E., Roman B., Marder M., Shin G.-S. and Swinney H. L., Nature, 419 (2002) 579.

[2] Audoly B. and Boudaoud A., Phys. Rev. Lett., 91 (2003) 086105.

[3] Sharon E., Roman B. and Swinney H. L., Phys. Rev. E, 75 (2007) 046211.

[4] Audoly B. and Boudaoud A., Comptes Rendus Mecanique, 330 (2002) 831.

[5] Marder M., Sharon E., Smith S. and Roman B., Europhys. Lett., 62 (2003) 498.

[6] Marder M., Foundations of Physics, 33 (2003) 1743.

[7] Liang H. and Mahadevan L., Proc. Nat. Acad. Sci., 106 (2009) 22049.

[8] Efrati E., Klein Y., Aharoni H. and Sharon E., Physica D: Nonlinear Phenomena, 235 (2007) 29.

[9] Klein Y., Efrati E. and Sharon E., Science, 315 (2007) 1116.

[10] Kim J., Hanna J. A., Byun M., Santangelo C. D. and Hayward R. C., Science, 335 (2012) 1201.

[11] Goriely A. and Amar M. B., Phys. Rev. Lett., 94 (2005) 198103.

[12] Ben Amar M. and Goriely A., J. Mech. Phys. Solids, 53 (2005) 2284.

[13] Ortiz M. and Gioia G., J. Mech. Phys. Solids, 42 (1994) 531.

[14] Ben Belgacem H., Conti S., DeSimone A. and Müller S., J. Nonlinear Sci., 10 (2000) 661.

[15] Bella P. and Kohn R. V., J. Nonlinear Sci., 24 (2014) 1147.

[16] Han Q. and Hong J.-X., Isometric embedding of Riemannian manifolds in Euclidean spaces (AMS, Providence, RI) 2006.

[17] Amsler M.-H., Mathematische Annalen, 130 (1955) 234.

[18] Efimov N. V., Matematicheskii Sbornik, 106 (1964) 286.

[19] Nechaev S. and Voituriez R., J. Phys. A: Mathematical and General, 34 (2001) 11069.

[20] Efrati E., Sharon E. and Kupferman R., J. Mech. Phys. Solids, 57 (2009) 762.

[21] Fung Y. C., Foundations of solid mechanics (PrenticeHall, Englewood Cliffs, N.J.) 1965.

[22] Lewicka M., Mahadevan L. and Pakzad M. R., Proc. Roy. Soc. London Ser. A, 467 (2011) 402.

[23] Lewicka M. and Reza Pakzad M., ESAIM: Control, Optimisation and Calculus of Variations, 17 (2011) 1158.

[24] Audoly B. and Pomeau Y., Elasticity and geometry: from hair curls to the non-linear response of shells (Oxford University Press Oxford) 2010.

[25] Gemmer J. A. and Venkataramani S. C., Physica D: Nonlinear Phenomena, 240 (2011) 1536.

[26] Bobenko A. I. and Pinkall U., Oxford lecture series in mathematics and its applications, 16 (1999) 3.

[27] Baker Jr G. A., Physical Review, 124 (1961) 768.

[28] Lobkovsky A., Gentges S., Li H., Morse D. and Witten T. A., Science, 270 (1995) 1482.

[29] Lobkovsky A. E., Phys. Rev. E., 53 (1996) 3750.

[30] Ben Amar M. and Pomeau Y., Proc. Roy. Soc. London Ser. A, 453 (1997) 729.

[31] Gemmer J. and Venkataramani S., Nonlinearity, 25 (2012) 3553 . 